\title{
SocArXiv
}

Social Science \& Medicine : 2018.07.26 doi.org/10.1016/j.socscimed.2018.09.005

\section{Validation of a New Continuous Geographic Isolation Scale: A Tool for Rural Health Disparities Research}

\author{
Nathan J. Doogan \\ The Ohio State University \\ Mary Ellen Wewers \\ The Ohio State University
}

Elizabeth A. Mumford

National Opinion Research Center

\author{
Megan E. Roberts \\ The Ohio State University
}

Erin R. Tanenbaum

National Opinion Research Center

Frances A. Stillman

National Opinion Research Center

\begin{abstract}
The purpose of this study was to develop and test a new continuous measure for rural health disparities research to characterize geographic areas according to a perspective of access to resources. We call the measure Isolation and anticipate it will be useful as an alternative to commonly used rural classification schemes (e.g., the Census Bureau's measure). Following the best known standards for measuring rurality, it captures the trade-off between access to resourcerich, high-population-density areas and the cost of travel to those areas; thus even intrinsically low-resource areas may have high access to nearby resources. Validity was tested with proxies such as nighttime outdoor lights, distance to hospitals, physician availability, and access to high quality food. The Isolation scale demonstrated good construct validity (i.e., both convergent and criterion validity). Fit statistics indicated that, compared to other commonly-used urban/rural definitions, the Isolation scale was the best overall measure when predicting several proxies for rurality, even when categorized. We also show that the measure does a substantially better job at explaining national health outcome data at the state level. This new continuous Isolation scale shows considerable promise for improving our conceptualization, theorization, and measurement of the features of rurality that are pertinent to rural health disparities research, and can also be useful to policy makers who may find value in using isolation thresholds that are most relevant to their policy planning needs.
\end{abstract}

Keywords: rural; resource access; health; geography; measurement.

\section{Acknowledgement}




\section{INTRODUCTION}

Inequitable rural access to health-relevant resources is a global issue of social and health justice (Scheil-Adlung 2015) that results in poorer health outcomes for people living in rural areas around the world (Humphreys and Solarsh 2008). An estimated 56\% of the global rural population lacks health coverage, compared with $22 \%$ of urban populations. Reasons for the inequity include, but are not limited to, poor infrastructure in rural areas and a severe disparity in the rural health workforce and in rural health spending. Moreover, the inequity is not limited to developing countries. Serious health disparities exist between the populations of US rural and urban areas, such that rural America has worse overall health outcomesfrom heart disease, to cancer, to infant mortality (Lengerich et al. 2005; Rural Health Reform Policy Research Center 2014; Singh et al. 2012). Many factors contribute to these disparities. On average, rural Americans have lower income, lower educational attainment, and reside in areas with fewer local health promoting policies and resources (Byun et al. 2012; Caldwell et al. 2016; McMillen et al. 2004; Northridge et al. 2008; US Department of Agriculture (USDA) Economic Research Service (ERS) 2017). Tobacco use is also a notable contributor to these disparities, as rural Americans are more likely to smoke cigarettes - the leading cause of preventable death - and use smokeless tobacco than their urban counterparts (American Lung Association 2012; Doogan et al. 2017; Roberts et al. 2016; Substance Abuse and Mental Health Services Administration 2013). If the existence of health inequity is itself an insufficient argument, consider also the extreme economic costs. One estimate of the cost of racial and ethnic health inequity to the US alone between the years 2003 and 2006 (in 2008 dollars) is well over one trillion dollars in preventable direct and indirect costs (LaVeist et al. 2011). Coupling this with well-documented rural health inequity, advancements in rural health research are not only beneficial to social and health justice, but also to the economy. The purpose of this article is to present an advancement in the measurement of what constitutes rurality according to our best understanding of the underlying causes of rural health disparities.

Existing measures that characterize US areas according to rurality have helped to identify many important geographic health disparities. However, the most commonly used measures in the US were not designed for health research. For example, common measures either do not give any attention to degrees of rurality (i.e., they dichotomously classify areas as urban or rural), or for those that do, the levels are defined according to factors with indirect relevance to health (e.g., economic commuting flows and potentially inappropriate classification thresholds). New measures are needed to facilitate simpler and clearer operationalization of what rurality is with respect to how it could relate to health. Such measures should enhance precision in the development and testing of specific geography related health hypotheses by ensuring researchers know what is measured and have the flexibility to choose thresholds, if they so desire, that make sense for their questions. With this contribution, we hope to facilitate further advancement of measurement as well as geography oriented health theory.

Health oriented measures of the urban-rural continuum have recently been developed in other nations, including Canada, Australia, and China, or for global use (Alasia et al. 2017; Humphreys et al. 2012; Li et al. 2015; Bank 2016), most of which are founded on health relevant factors such as access to resources that are likely to impact health outcomes. Discussion about potential revisions to common US measures are published (National Academies of Sciences, Engineering, and Medicine 2016), but we are currently unaware of general purpose US 
measures designed to capture - without arbitrary classification-isolation from urban centers, in which health relevant resources are most prominent. New measures specifically designed for health research, which give attention to degrees of rurality with a health-relevant basis, are warranted.

Most research on US urban/rural health disparities relies on a few established classification systems. Three definitions are most commonly used, and a fourth less commonly used definition also exists. First, the Census Bureau classifies census blocks as belonging to Urbanized Areas and Urbanized Clusters based primarily on population density but also nearness to regions of high density, with all remaining areas classified as Rural; we refer to this as the Census Bureau definition. Second, the Office of Management and Budget (OMB) classifies counties as Metropolitan, Micropolitan, or Non-Core ("rural") based on population density and proximity to adjacent urbanized areas according to commuting data; we refer to these as the OMB codes. And third, the US Department of Agriculture's Economic Research Service (USDA-ERS) classifies census tracts as Metropolitan, Micropolitan, Small Town, and Rural based on urban density and commuting flows in its scheme of Rural-Urban Commuting Areas (RUCAs). A fourth and less commonly used measure is the Frontier and Remote Area (FAR) Codes, also developed and maintained by the USDA-ERS (US Department of Agriculture (USDA) Economic Research Service (ERS) 2015b). FAR classifies census tracts (or zip codes, optionally) based on their population density and the travel time required to reach urban areas.

The common use of these definitions is encouraged by their frequent inclusion in large, national datasets. For example, the National Survey on Drug Use and Health provides data users with the urban/rural classification for each participant according to the OMB definition. Their common use is also facilitated by their public availability, which allows these variables to be easily merged with data sets that include geographic identifiers (e.g., county, census tract, or latitude and longitude information).

In our view, the primary limiting factor of all the discussed measures is in their prescription of what constitutes rurality (i.e., prescribed thresholds), which varies considerably depending on the measure. The Census Bureau's definition relies on a high threshold of population density to identify "urban," and fails to adequately delineate degrees of rurality. The measure tends to over-classify areas as rural, such that suburban and rural areas are often lumped together. The OMB definition does provide some sensitivity to degrees of rurality by incorporating adjacency to urban areas into its classification scheme; however, it only accounts for immediately adjacent urban-classified areas, which are so classified at the low-resolution county level. As a result, the OMB definition is often criticized for under-classifying areas as rural. Moreover, what qualifies as an urban-classified area is obfuscated by complicated and sometimes politically motivated or otherwise irrelevant rules (Morrill et al. 1999). The USDA-ERS developed RUCA codes as a reaction to some of these limitations. The system uses a census tract level of resolution, necessary for accurate classification, and commuting flows to represent influence from other tracts, which are not necessarily nearby. Nevertheless, the RUCA classification system is a classification system with prescribed classes and meanings that may not fit all research needs. Finally, USDA-ERS designed the FAR code system to classify very isolated census tracts into graded degrees of extreme rurality (frontier rural). While it has the high resolution of census tracts and gives special attention to high degrees of rural isolation, the FAR code's classification system lacks sensitivity at the metropolitan end of the continuum, which renders it a limited general-use measure. 
Underlying each of these classification systems is a latent continuous measure of the distance to a nearby populated area - the currently best understood way to characterize the rurality of geographic areas (National Academies of Sciences, Engineering, and Medicine 2016). However, by imposing a classification scheme, the developers of existing measures have limited the degree of nuance that is possible when using the measures. The purpose of this research is therefore to develop a reasonable continuous measure of rurality, validate it, and leave it to rural researchers and policy makers to decide how to use it. While we develop the measure for the US, its construction depends on simple data that are likely to be available in many countries around world. We present this new and relatively simple measure as a means for codifying a resource-isolation perspective of what constitutes the rurality of an area. The measure distinguishes isolated areas from resource-rich urban centers and nearby census tracts that can access urban resources with relative ease (i.e., adjacency matters, but so does non-adjacent proximity). And as a continuous scale, we designed the measure to be highly sensitive to degrees of isolation at both ends of the scale. While we do not prescribe a classification system for the new measure, we do recognize the importance of classification or categorization for some analyses and for the purpose of alleviating confidentiality concerns when the data are organized at the individual level. We therefore give attention to this in our analysis of the new measure. Our analyses are designed to validate this new Isolation measure, and compare it to the three most commonly-used urban/rural classification systems and the FAR codes. Our approach includes examination of the convergent validity of the new measure, along with two separate analyses examining its criterion validity. The latter will also provide a comparison of all the rurality measures in terms of their utility for explaining health-relevant resource access and health outcome data.

\section{METHODS}

\subsection{Development of the Isolation Measure}

\section{Overview of the measure and description of its calculation}

We designed the Isolation measure to characterize a geographic area according to a resident's access to resources. "Access" refers to geographic accessibility and can either comprise an area's intrinsic resources, or extrinsic resources in nearby areas. We conceptually define access very similarly to the "accessibility" dimension of the Theory of Access by Penchansky and Thomas (Penchansky and Thomas 1981). However, we do not strictly focus on health care, but more generally on health related resources, and we do not invoke other dimensions of the theory. "Resources" could be goods or services that humans need to survive and thrive, such as food, healthcare, or internet access.

In our approach, population density serves as the fundamental indicator of resources intrinsic to a geographic area. This is consistent with an economic perspective: resource capital is often invested in densely populated areas (e.g., numerous outlets of a grocery or coffee corporation in a single city center). Access to capital in rural areas is much more limited, as evidenced by the development of programs such as the Rural Business Investment Program (US Department of Agriculture Rural Development 2016b) and Rural Business Development Grants (US Department of Agriculture Rural Development 2016a) by the US Department 
of Agriculture Rural Development. Even with the explosion of internet shopping and parcel delivery services, parcel delivery hubs remain focused where populations are most dense, resulting in relatively higher costs for delivery to remote rural areas (e.g., delivery charges are tiered depending on remoteness) (FedEx 2018; S.J. Consulting Group, Inc. 2011).

The Isolation measure that we developed does not directly distinguish between intrinsic and extrinsic resources. Instead, it represents access to resources, whether intrinsic or extrinsic. We therefore limit references to resources per se, and instead refer to resource access, a reversal of which could be referred to as resource Isolation.

We use Census tracts as the fundamental areal unit. Tracts in the US vary in size such that they contain approximately 1,200 to 8,000 people, and ideally about 4000 people in each. The median size of a Census tract is 4.8 kilometers squared. Using the Census Bureau's rural classification system, the median geographic size of an entirely rural census tract is approximately 254 kilometers squared (approximately 16 by 16 kilometers, or the land area of a small to medium sized US city), and the median size of a tract in an urban area or urbanized cluster is 3.8 kilometers squared.

By our definition, a tract's resource access is equal to the maximum of either (1) its own intrinsic resources (population density), or (2) an adjacent tract's resource access that is discounted depending on the cost of travel between the two census tract centroids. Likewise, the resource access of the adjacent tract could be its own population density, or it could be its access to one of its adjacent tracts' resource access (discounted for travel cost), whichever is larger. This pattern can continue for virtually unlimited steps. Thus, while the calculation depends on direct adjacency, the high resource access in one tract, whether from intrinsic or extrinsic sources, diffuses outward, diminishing in value at each step due to travel cost, to tracts that are potentially multiple adjacency steps away.

We use an iterative algorithm to obtain an Isolation score for each Census tract. At the first iteration of the calculation, the intrinsic resource value (population density) of a tract is compared among the intrinsic resource values of all adjacent tracts minus a discount for the travel cost to obtain those resources, and the largest value is selected as the updated resource access of the tract. Importantly, the new resource access value is not added or averaged with the previous value, but rather it replaces the old value. At the start of the second iteration, some tracts now have this new value representing their access to a neighbor's resources, but which was discounted for travel cost. The same calculation is then repeated except it is now based on the new values (in some cases, resource access in the second iteration will still be equal to population density). This process is repeated until no more changes occur. In this way, the measure captures the accessibility of resources in resource-rich areas to residents of relatively resource-poor areas as a function of distance. An alternative perspective is that areas increasingly distant from high density population areas are decreasingly likely to be locations in which significant investments are made to make resources available locally (i.e., intrinsically). For example, grocery stores are most common where many shoppers reside. In sum, the measure is intended to capture the cost of travel to obtain distant resources as well as the likelihood that resources will be available locally due to the economic demand for them.

A simple resource scenario may be helpful to further clarify how the measure works. Consider three tracts, $A, B$, and $C$ such that $A$ and $B$ have low intrinsic resources and $A$ is the most isolated of the three. Tract $C$ is a metropolitan area with high intrinsic resources. The geographic arrangement is that $A$ is adjacent to $B, B$ is adjacent to $C$, and tracts $A$ and $C$ are not adjacent $(A-B-C)$. The resource access of the relatively isolated tract $A$ 
is entirely due to extrinsic resource access from tract $B$, and the resource access of $B$ is also extrinsic in nature due to the intrinsic resources of the adjacent metropolitan tract $C$. In this simple case, the resource access of tract $A$ ultimately represents access to the intrinsic resources of tract $C$ (the metro area), through tract $B$ (a resource-poor area adjacent to the metro area). Therefore, the value to tract $A$ of resources in $C$ should be discounted for the full travel cost between $C$ and $A$. Indeed, our measure is designed such that the travel cost to distant tracts (i.e., further away than an adjacent tract) is approximately a sum of the multiple adjacency travel costs along the way. The value of $C$ 's resources to $B$ is discounted for the travel cost between $C$ and $B$. The value to $A$ of $B$ 's resource access, which is already discounted because it is derived from $C$, is further discounted for the distance between $B$ and $A$. Thus, the discounting continues as adjacencies are traversed.

Given such a measure of resource access, it then needs to be transformed into a useful measure of Isolation. The first is a logarithmic transformation. Our rationale is based on a desirable interpretation of what a difference between two values means. Census tract population density provides an intuitive example; density is strongly positively skewed such that when considering density differences, it is more intuitive to consider percentage differences rather than absolute differences. For example, adding 1 person per square mile to a low density tract with 2 people per square mile (an absolute difference of 1 ) is a substantial increase (50\%), while adding 1 person per square mile to a tract with 100 people per square mile (also an absolute difference of 1 ) is a relatively meaningless increase (1\%). Thus, in the case of such a skewed distribution, a transformation to a percentage difference representation, like a logarithmic transformation, can be useful. A logarithmic transformation of density would ensure that a one unit change has approximately the same meaning in terms of percentage change at every point in the scale. Because the above described measure of resource access is a function of population density, it also has an extremely skewed distribution, and we therefore log transformed it (natural log). Conveniently, this results in an approximately normal distribution. We also reverse-scored resource access so that larger values represent more Isolation from resources. And finally, to ease interpretation, the score is shifted so that the minimum value of the scale is zero (representing the least amount of Isolation).

\section{Mathematical definition of the measure}

To capture this in a mathematical form, we first define a discount function to determine how much a neighboring tract's resource access should be discounted due to the distance between tracts. Practically, this is intended to reflect that the longer an individual would need to travel to obtain distant resources, the less valuable those resources are to the individual. The discount function should decrease the value of a neighbor's resource access as travel cost

increases. For travel cost, we use two different approaches. The first, which we call the Distance version, uses the great-circle distance between census tracts' geographic centroids, which roughly approximates the average travel distance between the tracts. The second, the Time version, is this same centroid distance divided by the speed of the fastest road connecting the two tracts, and is therefore an approximation of the travel time. In either case, we refer to the travel cost (measured either as distance or time) as $d_{i j}$, where $i$ and $j$ are indices referring to the focal tract and the neighbor tract, respectively. To implement a discount for distance, we define the following function:

$$
v(i, j)=a_{j} \delta^{d_{i j}}
$$


In English, we use an exponential decay of the value of the neighbor's resources $a_{j}$ with respect to distance. The value of a neighbor's resource access is a function $v$ of the constant distance $d_{i j}$ between tracts $i$ and $j$ and a parameter $\delta$, which is user defined between or including 0 and 1 , and which controls the relationship between distance and the total discount to the value of the neighbor's resource access. Values of $\delta$ that are closer to zero discount the value of resources in a neighbor tract $j$ more for a given centroid-to-centroid distance.

The calculation of each tract's Isolation score involves each tract being assigned the maximum value among the evaluations of $v(i, j)$ for each neighboring tract $j$ including the case in which $j=i$ (i.e., effectively $v(i, i)$, which, because of the zero distance between $i$ and itself, simply equates to the current resource access of tract $i$ ). The decision is captured in the equation below defining the access score of any tract indexed by $i$ as the maximum across all neighbor-evaluations indexed by $j$ :

$$
a_{i}=\max _{j}[v(i, j)]
$$

Because of the dependency of a tract's resource access on its neighbors' resource access, the values of $a$ are calculated through an iterative process. We simply re-evaluate $a_{i}$ for all tracts repeatedly, each time inputting the last iteration values to represent $a_{j}$, until no tract's resource access value is updated from the last iteration. Final values will vary depending on the choice of $\delta$ (hypothetical examples of the measure for various values of $\delta$ are provided in Figure 1) that must be selected prior to calculation. Once again, we log transform, reverse (multiply by -1), and shift the result so that the minimum value is zero to obtain our final measure of Isolation.

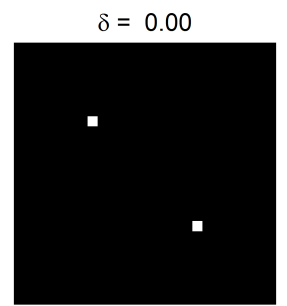

$\delta=0.75$

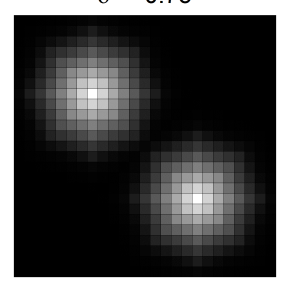

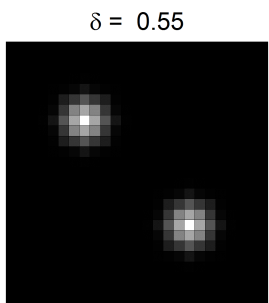

$\delta=0.85$

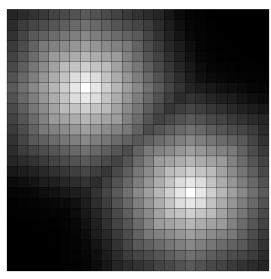

Figure 1: Demonstration of the result of the procedure in a controlled environment. Assume we have a 25 by 25 grid of one $\mathrm{km}$ by one $\mathrm{km}$ square census tracts $(25 \times 25=625$ tracts in total $)$. Within this grid, the population density of all census tracts except two is zero. The two census tracts with non-zero density each have a large population of one million (a density of one million per square kilometer). The panels depict the Isolation of the 625 tracts for four different values of $\delta$, with black areas being the most isolated. 


\subsection{Choosing a Value for $\delta$}

As previously stated, $\delta$ must be chosen by the researcher. Ideally, variability in the calculated Isolation score should map optimally onto actual measures of resource isolation (or access) so that the measure can reflect what we believe is the fundamental cause of health disparities related to rurality. We therefore calibrated the measure via $\delta$ to optimize the statistical fit between the Isolation score and several geographic outcome measures that indicate resource access (measures described below). We modeled each outcome measure as a function of the Isolation score 100 times, each time using a different version of the Isolation score created using a different value of $\delta$ ( 0 through .99 by increments of .01). Note that when $\delta$ equals zero, Isolation is perfectly negatively correlated with the log of population density. We chose an appropriate regression model for each resource measure - a log-normal regression for a distance measure and a rate measure, a logistic regression for a dichotomous indicator, and an ordinal regression for an ordinal measure. We then chose the value of $\delta$ that maximized the log-likelihood of the regression model for each resource. This value was considered the optimal $\delta$ for the given resource measure, and it varied by resource measure; to choose a suitable overall value of $\delta$, we averaged the individual optimal values (reported among the results), arriving at a final general-use value for $\delta$. This procedure was repeated for both the Distance and Time versions of the Isolation score. Optimization of $\delta$ is for calibration purposes only, and we assign no special meaning to the optimal value for $\delta$.

\subsection{Measures}

\section{Commonly Used Urban/Rural Measures}

Data for the three commonly-used urban-rural definitions were obtained from their respective agency's website, and the most recently-available year of classification was selected (2010 for the RUCA, 2013 for the OMB, and 2010 for the Census Bureau). Census Bureau and RUCA data were organized at the Census Tract level. OMB codes were organized at the county level. We also used the 2010 FAR code tract-level classifications. The five levels of the FAR code system range from 0 (no FAR designation) to 4 (most restrictive FAR designation; i.e., most rural).

\section{Measures Correlated with Resource Accessibility}

The measures below include those that directly measures access to resources and are used for calibration of the Isolation measure (via its $\delta$ parameter) and for fit checking relative to other measures of rurality.

Physician ratio is the number of physicians per 100,000 residents at the county level. The physician count included all federal and non-federal active MDs in 2010, and was divided by the size of the county population in 2010 according to the Census Bureau, then multiplied by 100,000. The physician ratio data were drawn from the Area Health Resource Files from the Health Resources \& Services Administration at the US Department of Health and Human Services (USDHHS) (US Department of Health and Human Services 2016).

Hospital distance is the distance of a tract's centroid to the nearest hospital, defined as an institution that provides medical or surgical treatment and nursing care for sick or injured people. Data were sourced from USDHHS, but stored and made available for download by Esri, a supplier of geographic information systems software (Esri 2017). 
Food access is a tract-level indication that at least $33 \%$ of the population, or 500 people, reside greater than 10 miles from a supermarket, supercenter, or large grocery store. The same or similar measures have been used in US health research including studies of food deserts (Liese et al. 2014) and shopping behavior depending on food accessibility (Sohi et al. 2014). Data were made available by the USDA-ERS (US Department of Agriculture (USDA) Economic Research Service (ERS) 2015a).

Internet service is a six-level ordinal measure of the rate of residential fixed high-speed internet connection subscriptions per 1,000 households in each census tract. Data were obtained from the Federal Communications Comission (Federal Communications Commission 2015).

\section{Additional Variables Correlated with Rurality for Validation}

Two other measures were obtained that address rurality, but do not directly represent resource access. These are therefore not used for optimization of the $\delta$ parameter. These measures are likely best at characterizing where resources are, rather than how much access to resources an area has. Therefore, we expect these measures to be correlated with Isolation and other rural measures, but less so with those rural measures that appropriately characterize distance to resources.

Nighttime lights represents satellite-obtained low light imaging data of the US at night and includes light generated by any source on the surface, including electricity and fire. The data exclude glare and light from the aurora or reflected from the moon, and observations are averaged over a full year (2010) of night-time observations. The data were obtained from the National Oceanic and Atmospheric Administration National Geophysical Data Center (National Oceanic and Atmospheric Administration 2010). Their current system combines data captured at low gain and high gain settings, to produce a global nighttime lights product with high sensitivity to light intensity. Resolution was reduced to 300-by-300 meter readings (for computational feasibility), which were averaged within each Census tract to result in a tract-level light intensity measure.

Land cover quantifies the portion of land in a Census Tract that has surface features that render it "developed" land. Data were obtained from the Multi-Resolution Land Characteristics Consortium (Multi-Resolution Land Characteristics Consortium 2014), which provides ecosystem classifications for all US land cover. Four classifications were considered developed: Developed, Open Space; Developed, Low Intensity; Developed, Medium Intensity; Developed, High Intensity. All other classifications (e.g., Barren Land, Deciduous Forest, Cultivated Crops) were coded as "undeveloped."

\section{Road Network Data}

We obtained data about the road network and the speed of roads from OpenStreetMaps (OpenStreetMap contributors 2017). These data originated from the US Census Bureau in 2005 , but were heavily augmented by volunteers who incorporate additional useful information about road and other land features, including the speed limit of roads and the type of roads (e.g., motorway, trunk, primary, secondary, residential) for individual road segments. Only a fraction of road segments included speed limit data. However, virtually all of them are defined with a road type. We therefore calculated an overall average speed limit for each type of road, and then imputed road speeds based on road type for all road segments with a missing road speed. 


\subsection{Analyses}

Upon selecting a general-use value for $\delta$ and calculating the final Isolation scale scores for the Distance and Time versions separately, the remaining analyses consisted of assessing the Isolation scale's construct validity. Specifically, tests for construct validity assessed the Isolation scale's convergent and criterion validity.

For convergent validity, a scale should be correlated with measures to which it is theoretically related - in this case, the other urban/rural measures. Therefore, we ran a series of Spearman correlations to test whether both versions of the Isolation scale were significantly related to the three commonly-used measures of urban/rural plus the FAR codes.

For criterion validity, a scale should be related to an outcome; it is typically assessed by comparing the scale's agreement with a gold standard measurement (DeVellis 2011). As noted above, there is no gold standard measure of true rurality with which to validate our Isolation measure. Therefore, as a first step, we used our various dependent measures as proxies for rurality: physician ratio, distance to hospitals, food access, and internet service. This analysis also included an examination of nighttime lights and land cover, which we expect to be correlated with Isolation, but recognize they measure something subtly different than resource access (i.e., we expect Isolation to relatively poorly predict lights and land cover). Each dependent measure was regressed on each urban/rural measure (Isolation, RUCA, OMB, Census Bureau, and FAR). We then statistically compared the fit of the urban/rural definitions to determine which best explained the variation in each dependent measure. Specifically, regression models were ranked according to the Akaike information criterion (AIC) (Burnham and Anderson 2002), a likelihood-based measure of relative fit. We use AIC, as opposed to the likelihood itself, because the models in the comparison vary in complexity (i.e., the number of parameters, which is a function of the number of levels of the rural measures), and AIC penalizes models with higher complexity to strike a balance between model/measure complexity and fit.

Whether Isolation can at least equally explain real health outcome measures should also be of interest to health researchers. We therefore additionally examined the relationship between state-level population exposure to rurality, as defined by each rurality measure, and three state-level health outcomes. Population exposure to rurality is calculated as the proportion of the population that a given measure characterizes as rural. For the two versions of Isolation, we calculated the proportion of the population exposed to levels of Isolation that exceeded the national upper quartile of Isolation. This is an arbitrary choice, and researchers could justify their own threshold choice. The first outcome is the infant mortality rate, which has been shown empirically to be higher in rural areas (Ely et al. 2017). Likewise, the second is smoking related mortality rate, which theoretically should be higher in rural areas due to an elevated prevalence of smoking (Doogan et al. 2017). Finally, we examine smoking quit ratios, which are defined as the proportion of former smokers among ever smokers. A US study has shown that rural residents in West Virginia find it more difficult to quit than their urban counterparts (a likely partial explanation for higher overall prevalence in rural areas) (Northridge et al. 2008). The infant mortality data were obtained from the CDC WONDER online database US Centers for Disease Control and Prevention (CDC) (2016). The quit ratio data and the smoking related mortality data were obtained from the Centers for Disease Control (CDC) State Tobacco Activities Tracking and Evaluation (STATE) System.Centers for Disease Control and Prevention (2018). 
The health outcome analysis involves the calculation of one regression model per outcome per rurality exposure predictor (18 models in total). In all cases, an ordinary least squares approach is used because the outcome data appear to have a normal distribution. The analysis provides two important pieces of information. First, it will reveal whether Isolation, and other rurality measures, can identify these known or expected relationships. Second, the analysis can reveal, via examination of a variance-explained metric $\left(R^{2}\right)$, which measure best explains the geographic patterns of these health outcomes.

The above statistical analyses included a total of four versions of the Isolation score (not all used in every analysis). These include both the Distance and Time versions as previously described, as well as categorical versions of both. For clarity, we descriptively refer to the measure variations as Isolation-Distance-continuous, Isolation-Distance-categorical, Isolation-Time-continuous, and Isolation-Time-categorical. The categorical versions allowed us to make a fairer comparison of Isolation with other measures of rurality that are categorical, and to give one example of how the measure can be categorized. We used a four-level categorization, which is the maximum number of levels among the other measures assessed in this study. Moreover, if Isolation were to be included in a public national individual-level dataset, it would very likely not be included in its continuous form due to confidentiality concerns. We thus use both versions in our analyses to support an understanding of what might be lost in categorization. In the health resource outcome models, the rural predictor variables were coded as sets of dummy variables for all but one of their levels, except for the continuous versions of Isolation which were treated as continuous variables. To evaluate sensitivity to categorization, we also briefly report changes in rank when Isolation-\{Distance,Time $\}$ categorical is categorized into three- and five-level versions. Importantly, our choice of the number of categorization levels is based on validation analysis concerns. In practical use, versions with more levels will conserve more information about resource isolation, and should be considered for use in lieu of a four level version, to the extent possible.

\section{RESULTS}

\subsection{Descriptive Statistics for the Isolation Scale}

The calculation procedure for both versions of the Isolation scale required between one (for $\delta=0$ ) and 97 (for $\delta=.99$ ) iterations, with higher values of $\delta$ requiring more iterations due to a longer diffusion of resources associated with the lower travel-cost discount.

For Isolation-Distance-continuous, the average of the optimal values of $\delta$ representing the best fit for each resource measure (physicians: 0.91 ; hospitals: 0.73 ; food: 0.83 ; internet: 0.98), and thus the general-use value to be used in the present analyses, was 0.86. For Isolation-Time-continuous, the average of the optimal values of $\delta$ representing the best fit for each resource measure (physicians: 0.93; hospitals: 0.78; food: 0.86; internet: 0.98), and thus the general-use value to be used in the present analyses, was 0.89 . We report the optimal value of $\delta$ for posterity only and place no special meaning on it. The categorical versions of the measures were derived from the continuous versions by cutting them at their quartiles. 
The Isolation-Distance-continuous scale had a mean of $5.2(S D=2.0$, range $0-12.1)$. The 25th (lower quartile), 50th (median), and 75th (upper quartile) unweighted percentiles were 4.0, 4.8, and 6.1, respectively. The Isolation-Time-continuous scale was very similar with a mean of $5.3(S D=2.1$, range $0-12.1)$. The 25 th (lower quartile), 50 th (median), and 75th (upper quartile) unweighted percentiles were 4.0, 4.8, and 6.2, respectively.

These values are not particularly meaningful per se, but interpretation can be intuitive when considering score differences between areas. For example, because the measure is intended to capture isolation from health relevant resources, and because the scale is log transformed using the natural logarithm (base $e$ ), then an $x$ unit increase in Isolation score can be thought of as a multiplicative change in isolation from resources by a factor of $e^{x}$ (approximately $2.72^{x}$ ). Alternatively, it could be interpreted as a multiplicative change in resource access (the reverse of resource isolation) by a factor of $e^{-x}$.

Figure 2 illustrates Isolation-Distance-continuous scale scores mapped across the entire US. The map shows white, high resource points at city centers and smooth transitions out to darker, more isolated areas.

As an example of how tracts were scored by the Isolation-Distance-continuous version of the measure, tract \#36061007600 in the well-known urban area of Manhattan, New York City, had an Isolation score of 1.2, one of the least isolated areas in the country. Tract \#06085511608 in what would be considered a suburban area in Stanford, CA, had an Isolation score of 4.0, approaching the median of the Isolation distribution. Comparatively, this amounts to approximately 16 times the isolation, or about $6 \%$ of the resource accessibility of an individual living in Manhattan. Tract \#06071010300, which is in the middle of the Mojave Desert, had an Isolation score of 11.9, one of the most isolated areas of the country by our measure.

\subsection{Construct Validity}

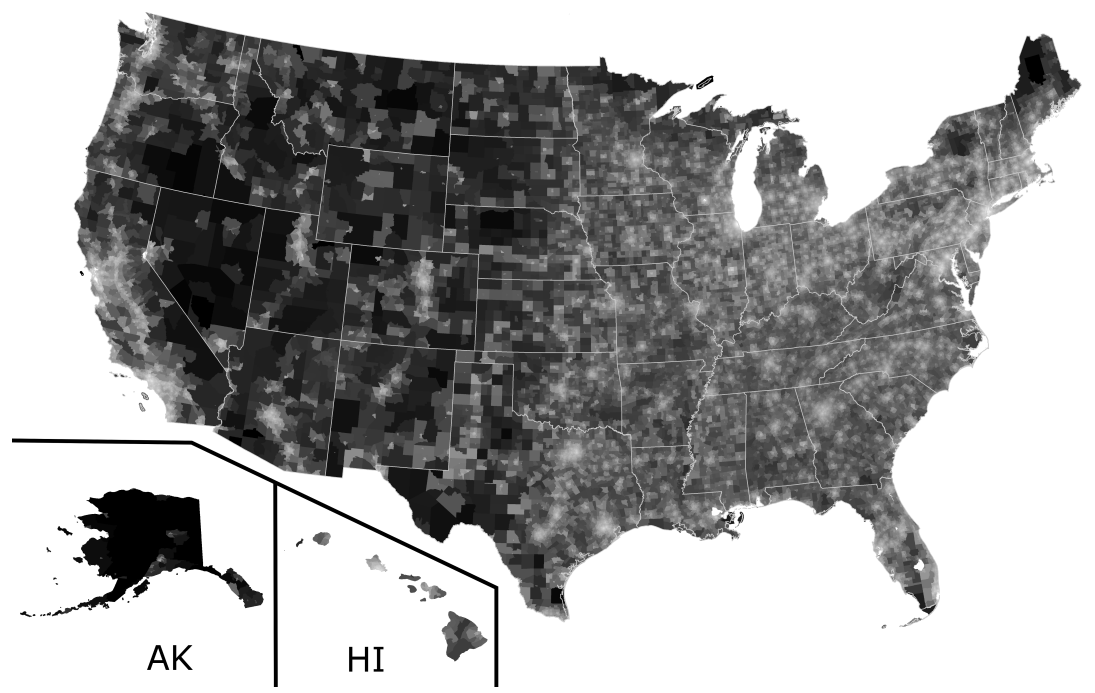

Figure 2: Geographic visualization of the Isolation-distancecontinuous scale $(\delta=0.86)$ across the US. Darker areas indicate greater Isolation. 
Table 1: Correlations among the Urban/Rural measures, and percentage of the population that each definition classifies as rural. The Isolation scale included is the continuous version.

\begin{tabular}{lccccc}
\hline \multicolumn{1}{c}{ Measure } & 2. & 3. & 4. & 5. & 6. \\
\hline 1. Isolation-Distance & 0.99 & 0.54 & 0.54 & 0.78 & 0.30 \\
2. Isolation-Time & & 0.54 & 0.53 & 0.78 & 0.30 \\
2. RUCA & & & 0.81 & 0.50 & 0.49 \\
4. OMB & & & 0.50 & 0.51 \\
5. Census Bureau & & & 0.28 \\
6. FAR & & & \\
\hline Note: All correlations are significant at p <.05 \\
RUCA = Rural-Urban Commuting Areas \\
OMB = Office of Management and Budget \\
FAR = Frontier and Remote Areas
\end{tabular}

Table 1 presents an unweighted correlation matrix indicating that the Isolation measure is significantly related to all other existing urban/rural measures. These findings support the convergent validity of the Isolation measure. Notably, the Distance and Time versions of Isolation are very similar, though not identical $(\mathrm{r}=0.99)$.

Table 2 presents the fit statistics for each of the outcome variables - nighttime lights, land cover, physician ratio, distance to hospitals, food access, and internet access - regressed on each of the rural measures including four different versions of the Isolation score - the categorical and continuous versions of both the Distance and Time versions. Rankings of the AIC relative fit statistic values indicate that some version of the Isolation measure is the best at explaining all outcomes except for land-cover, which was not expected to be a particularly meaningful measure of resource accessibility. Moreover, in the case of most outcomes, the various versions of Isolation dominate the highest ranks. Among the Isolation scales, Isolation-Distance-continuous sticks out as being particularly good at explaining the resource access measures. And while it is clear that the two categorical versions of Isolation involve some loss of information, they still tend to rise above the other measures of rurality in terms of explaining these resource access measures. An improvement of between 10 and 20 (towards a more negative value) from one AIC value to another is generally thought to be very substantial (Burnham and Anderson 2002); in the case of the present analyses, all differences between the fit of the Isolation scale and the other measures should thus be considered very substantial. These results support the criterion validity of the Isolation measure, as well as the superiority of Isolation in terms of characterizing isolation from health related resources.

When we categorized both versions of Isolation into 5-category variables, the AIC values for both became substantially better (not reported in the table). Most of the rankings stayed the same, except that where the Census Bureau measure better explained food access than both categorical versions of Isolation in the 4-level measure, the 5-level measure overcame that weakness. When we categorized both into 3-level variables, the ranking of Isolation became much worse for most outcomes, such that the Isolation scales no longer completely dominated the rankings for explaining health resource outcomes. 
Finally, table 3 presents the results of the 18 regression models of health outcomes on population exposure to rurality, as characterized by each rural measure. The results indicate that in the case of all three outcomes, population exposure as characterized by the two versions of Isolation is a significant predictor of the outcome in the expected direction $(p<.05)$. The same cannot be said, uniformly across outcomes, for any of the other measures. Additionally, the reported $R^{2}$ values further separate Isolation from the other measures in terms of how well they explain the geographic patterns of the examined health outcome variables. Particularly impressive are the comparisons between the $R^{2}$ values of both versions of Isolation and all other measures with respect to infant mortality (Isolation-Distance $R^{2}=0.17$, Isolation-Time $R^{2}=0.18$, maximum $R^{2}$ of all others was 0.03 ) and smoking quit ratios (Isolation-Distance $R^{2}=0.25$, Isolation-Time $R^{2}=0.26$, maximum $R^{2}$ of all others was 0.15 ). The dominance of Isolation for the smoking related mortality rate outcome is also notable, but more moderate (Isolation-Distance $R^{2}=0.56$, Isolation-Time $R^{2}=0.56$, maximum $R^{2}$ of all others was 0.53). Finally, while the analyses of health related resource outcomes seemed to indicate that Isolation-Distance was ranked highest, the $R^{2}$ values in this analysis very slightly point to Isolation-Time as a superior measure for health outcomes given this particular analysis.

Table 2: Fit statistic outcomes for the urban/rural measures regressed on nighttime lights, land cover, physician ratio, distance to hospitals, food access, and internet access. For each outcome, urban/rural measures are also ranked from $1=$ best fit, $5=$ worst fit.

\begin{tabular}{|c|c|c|c|c|c|c|c|c|c|c|c|c|}
\hline & \multicolumn{2}{|c|}{ Night Lights } & \multicolumn{2}{|c|}{ Land Cover } & \multicolumn{2}{|c|}{ Physicians } & \multicolumn{2}{|c|}{ Hospitals } & \multicolumn{2}{|c|}{ Food Access } & \multicolumn{2}{|c|}{ Internet Service } \\
\hline & $\mathrm{AIC}$ & Rank & $\mathrm{AIC}$ & Rank & $\mathrm{AIC}$ & Rank & $\mathrm{AIC}$ & Rank & $\mathrm{AIC}$ & Rank & $\mathrm{AIC}$ & Rank \\
\hline Isolation-Distance-continuous & 143343 & 2 & -9413 & 5 & 162937 & 1 & 175360 & 1 & 12797 & 1 & 151786 & 4 \\
\hline Isolation-Distance-categorical & 173079 & 4 & -21739 & 3 & 168543 & 4 & 177820 & 3 & 16730 & 4 & 151494 & 1 \\
\hline Isolation-Time-continuous & 142652 & 1 & -10057 & 4 & 162960 & 2 & 175560 & 2 & 12948 & 2 & 151774 & 3 \\
\hline Isolation-Time-categorical & 173051 & 3 & -22256 & 2 & 168467 & 3 & 178004 & 4 & 16901 & 5 & 151625 & 2 \\
\hline RUCA & 228719 & 6 & 38884 & 6 & 174250 & 7 & 196959 & 6 & 20464 & 6 & 152196 & 6 \\
\hline OMB & 230637 & 7 & 40184 & 7 & 168579 & 5 & 197273 & 7 & 20465 & 7 & 152192 & 5 \\
\hline Census Bureau & 180230 & 5 & -39497 & 1 & 171378 & 6 & 183037 & 5 & 15995 & 3 & 152474 & 7 \\
\hline FAR & 248267 & 8 & 53514 & 8 & 185104 & 8 & 200933 & 8 & 22205 & 8 & 157651 & 8 \\
\hline
\end{tabular}

RUCA $=$ Rural-Urban Commuting Areas

$\mathrm{OMB}=$ Office of Management and Budget

$\mathrm{FAR}=$ Frontier and Remote Areas

\section{DISCUSSION}

This study presents a new urban/rural measure-Isolation - that balances precision and practicality when identifying rural areas according to their isolation from health related resources. The measure essentially captures the trade-off between access to resource-rich, highpopulation-density areas and the cost of travel to those areas; or alternatively, it captures the likelihood that resources will be placed in a given area as a result of the number of people who could and would reasonably access it (i.e., demand). In doing so, the measure, like most other accepted definitions of rurality, incorporates both population density and travel costs to reach densely populated areas into its calculation. Theoretically, the measure can be seen as a proxy for a constellation of health risk factors that are correlated with proximity to population centers, such as health care, quality food, and information (e.g., via high-speed internet connections). We developed and examined the measure for use in US health research. How- 
Table 3: Coefficients, standard errors, and $R^{2}$ values for regression models of state-level infant mortality rate, smoking related mortality rate, and smoking quit ratios. The table presents the results of six regression models for each outcome.

\begin{tabular}{|c|c|c|c|c|c|c|c|c|c|}
\hline \multirow[t]{3}{*}{ Rurality Measure } & \multicolumn{9}{|c|}{ Health Outcome Measure } \\
\hline & \multicolumn{3}{|c|}{$\begin{array}{l}\text { Infant Mortality } \\
\text { Rate }\end{array}$} & \multicolumn{3}{|c|}{$\begin{array}{l}\text { Smoking Related } \\
\text { Mortality Rate }^{\dagger}\end{array}$} & \multicolumn{3}{|c|}{ Smoking Quit Ratios } \\
\hline & $\begin{array}{l}\text { Rurality } \\
\text { Coef. }\end{array}$ & $S E$ & $R^{2}$ & $\begin{array}{l}\text { Rurality } \\
\text { Coef. }\end{array}$ & $S E$ & $R^{2}$ & $\begin{array}{l}\text { Rurality } \\
\text { Coef. }\end{array}$ & $S E$ & $R^{2}$ \\
\hline Isolation Distance & $0.03^{*}$ & $(0.01)$ & 0.17 & $0.53^{*}$ & $(0.18)$ & 0.56 & $-0.17^{*}$ & $(0.04)$ & 0.25 \\
\hline Isolation-Time & $0.03^{*}$ & $(0.01)$ & 0.18 & $0.54^{*}$ & $(0.18)$ & 0.56 & $-0.18^{*}$ & $(0.04)$ & 0.26 \\
\hline RUCA & -0.01 & $(0.02)$ & 0.00 & 0.21 & $(0.30)$ & 0.48 & -0.09 & $(0.08)$ & 0.03 \\
\hline OMB & 0.01 & $(0.01)$ & 0.03 & $0.55^{*}$ & $(0.24)$ & 0.53 & $-0.18^{*}$ & $(0.06)$ & 0.15 \\
\hline Census Bureau & 0.02 & $(0.02)$ & 0.03 & $0.78^{*}$ & $(0.33)$ & 0.52 & $-0.19^{*}$ & $(0.08)$ & 0.09 \\
\hline FAR & -0.01 & $(0.01)$ & 0.00 & -0.14 & $(0.25)$ & 0.48 & -0.08 & $(0.06)$ & 0.03 \\
\hline $\begin{array}{l}\text { RUCA = Rural-Urban } \\
\text { OMB = Office of Man } \\
\text { FAR = Frontier and R } \\
* \text { Statistically significa }\end{array}$ & $\begin{array}{l}\text { Commutir } \\
\text { gement an } \\
\text { mote Are } \\
\text { it at the le }\end{array}$ & $\begin{array}{l}\text { Areas } \\
\text { d Budget } \\
\text { s } \\
\text { vel of } p<\end{array}$ & & & & & & & \\
\hline
\end{tabular}

ever, the components of the measure (population density of well-defined areas and estimates of travel cost among them) are neither complicated, nor difficult to obtain for many countries around the globe, and are consistent with important fundamental factors cited and used in recently developed measures of rural resource access in other countries (Alasia et al. 2017; Humphreys et al. 2012). Our results suggest that Isolation is clearly at least as good as, and likely better than, existing commonly used rural classification systems for explaining health related measures (i.e., resource access and health outcomes). Aside from predictive superiority demonstrated by our results, the measure stands as a general purpose continuous measure of what many experts agree constitutes rurality (National Academies of Sciences, Engineering, and Medicine 2016) with no prescription about which threshold most validly represents the distinction between urban and rural areas. Thus, Isolation is a new and flexible tool for rural policy makers and health researchers to use in their research and build upon.

The Isolation scale demonstrated good construct validity (i.e., both convergent and criterion validity). The distribution of the scale, as well as its visualization in Figures 1 and 2 also demonstrate the sensitivity of the Isolation scale to gradients of rurality. Furthermore, findings indicated that the continuous version of the Isolation scale was the best overall measure when predicting various proxies for rurality that included several measures of access to specific health related resources. Specifically, the Isolation scale, in almost all of the forms we examined, performed better than all the other commonly-used urban/rural classification systems when predicting physicians, hospitals, food access, and internet service. Perhaps most striking was the Isolation scale's dominance in explaining state-level variation in three important health outcomes: infant mortality rate, smoking related mortality rate, and smoking quit ratios. The results of these analyses provide very compelling evidence that the Isolation scale consistently captures aspects of health-relevant resource access that other typical measures of rurality used in the US do not. We expect these findings are more a 
function of prescribed classification thresholds, rather than a difference in the latent measure of distance from densely populated urban centers that characterizes all of the commonly used measures of rurality. Our measure, which excludes prescriptive thresholds, therefore stands as an important contribution to the effort to eliminate rural health disparities.

A notable deviation from the high performance of the Isolation scale is that no form of the measure was the best for explaining land cover data; the Census Bureau measure was better by a large margin. Interestingly, though, the Census Bureau measure was comparatively weak at explaining the distribution of internet service. As the creation of the Isolation scale is premised on the concept of access to resources, the fact that our measure performed better when predicting resource-based measures (e.g., medical care, food access, internet service) than land cover is neither surprising nor concerning. Our results therefore suggest that the Isolation measure, with a focus on access to goods and services relevant to well-being, has promising potential for geographically related health research.

Our analysis of resource accessibility outcomes included 4-category versions of the Isolation scale for comparison with the continuous versions and to the other rurality measures. We found that despite some loss of information due to categorization, these versions still performed better than the commonly used measures - OMB, RUCA, Census Bureau, and FAR. As expected, a follow-up analysis that included a 5-level categorization of Isolation showed that this version was a substantial improvement over the 4-level version. A 3-level version showed a substantial decline in performance. Thus, it is clear that categorization incurs costs in the form of important information about resource isolation. Researchers, policy makers, and survey developers interested in using a categorical version of the Isolation scale must carefully consider the confidentiality-precision tradeoff when deciding on how to categorize the measure. Based on our analyses, and if using quantiles to generate equally sized groups, we recommend using a 4-level categorization, at the least. Five-level categorization or more is preferred to the extent that it is feasible. However, creating equally sized groups based on quantiles is only one of many ways to divide the population. Knowledge of the specific domain under study should inform how users choose to categorize the measure.

Our analyses assessed two different versions of the Isolation scale - the Distance version, and the Time version that modified the Distance version by incorporating the presence and speed of travel routes. Comparatively, the two measures performed almost identically. The simpler Distance version seemed to have a slight advantage for explaining resource access measures, while the Time version had a slight advantage explaining health outcome data. Given that superiority is mixed depending on the outcome, and is very subtle regardless, our conclusion is that the simpler measure is the more practical one. The small boost in varianceexplained seen for two of the three health outcomes does not, in our opinion, justify the additional effort required to incorporate travel route connectivity and speed data. However, the additional effort could have a different effect on explanatory power in other countries, perhaps where road quality is more variable, or with different research questions. Moreover, other methods of incorporating travel route and speed data that we did not attempt may provide more impressive boosts in explanatory power in the US.

Use of the principled, simple, and continuous Isolation measure can assist researchers and policy experts to address health disparities. Specifically, a sound methodological approach for identifying degrees of rurality, particularly as they pertain to access to health related resources, will be valuable to examining possible underlying causes of health disparities. As a basic example, the main effect of Isolation on a particular health outcome could be interesting, per se, in that it represents the effect of a very general form of isolation. However, the measure 
also opens the door to more careful research of specific types of isolation, or the ways in which isolation may interact with other exposures or behaviors. For instance, a researcher may be interested in studying the effect of precisely-measured food access on health. In this case, Isolation may be used to control for a general form of isolation so that the effect of a more specific form, food access, can be uniquely identified. Or perhaps the objective could be to learn to what degree specific types of isolation (e.g., medical services, information, food access) explain away an effect of general isolation as measured by the Isolation scale (as in mediation analysis). As a final example, intervention research could utilize the Isolation scale to understand how the effect of a behavioral intervention, such as a smoking cessation trial, is moderated by the level of isolation to which participants are exposed in their daily lives. With its sensitivity to gradients of rurality, the Isolation scale is well-suited to precise and nuanced identification of the risk factors that are unique to rural areas.

Improvement in the measurement of what constitutes geographic isolation can also help with targeting program services, interventions, health communication approaches, and policy implementation. For example, Humphreys and colleagues point to the inadequacy of current classification schemes in Australia to maximize among-group heterogeneity and within-group homogeneity (i.e., misclassification) (Humphreys et al. 2012). If workforce incentives are based on a classification scheme that has a high rate of misclassification, then healthcare spending on incentives will largely be concentrated in relatively high resource areas that happen to be misclassified as low resource areas, and the constituents of true low resource areas will not see their fair share. Our results suggest that Isolation is likely to be a substantial improvement on existing US measures in this regard, as it more directly represents resource isolation and better explains actual health related resource distributions and health outcomes that are hypothesized, and in some cases known to be, associated with geographic isolation from resources. The "best" measure of the urban-rural continuum depends on the research question or activity at hand and possibly also on the geography of the region under study. The Isolation scale was developed primarily for the purpose of rural health research and policy planning, and it is meant to reflect the isolating features thought to contribute to both rural culture and rural health outcomes - from low population density, to remoteness and isolation from resources (Hart et al. 2005; Hartley 2004; Cromartie and Bucholtz 2008). Yet, other topics may be better addressed with alternative measures. Therefore, we do not recommend disregarding other measures of rurality, and further examinations and comparisons are warranted, including with measures developed for use in other nations (Alasia et al. 2017; Humphreys et al. 2012; Li et al. 2015; Bank 2016).

The geographic distribution of people and resources is likely to continue changing over time. The Isolation scale's delta parameter and the simplicity with which the scores are calculated allows it the flexibility to adapt to such changes when recalculated on updated population density and resource data.

A limitation to the Isolation scale is that it assesses distance in terms of great-circle distance between tract centroids on a sphere; in other words, distances are measured as the crow flies. We settled on this approach due to its simplicity for calculation. It minimizes the data we need to have available to calculate the measure, and according to our analyses, appears to contribute to a better approximation of isolation from resources than other US measures of the urban-rural continuum, and it also better explains more distal health outcomes. Moreover, the approach we took to incorporating road network data to refine the distance measure such that it represents time did not appear to have an appreciable effect on the results of our analysis. We therefore recommend use of the simpler distance version. 
A worthwhile note, and what could possibly constitute a limitation, relates to the use of the maximum function (as opposed to an average or a weighted sum, etc.) in the mathematical formula underlying the scale. By definition, the calculation of the scale does not recognize the potential benefits that may result from agglomeration economies arising out of multiple (two or more) large and proximal urban centers. The maximum function dictates that only one source of resources - the one providing the highest value, accounting for travel distancedefines the resource access of a given tract, as opposed to some combination of all nearby tracts. Alternative mathematical definitions are possible and should be explored to the extent that potential limitations like this create concern.

It is also important to note that rural America is not homogenous. A tract with an Isolation score of 7.0 in West Virginia will not necessarily share the same culture and healthrisk factors as a tract with the same score in Montana. Other dimensions are likely to play important roles in health risk. Therefore, while Isolation can identify large scale differences in the distribution of isolation in different regions, on smaller scales (e.g., census tracts) it is a general measure of isolation that cannot parse apart, for example, cultural differences due to demographic factors that played no role in its development. Thus, researchers must take care to incorporate these important factors in their own analyses.

With such diverse geography in the US, defining "rural" is a challenge. The Isolation scale presented in this study provides a continuous measure that is highly sensitive to degrees of rurality by assessing increasing remoteness from high population density areas. Gaining a better understanding of the issues related to rural health disparities is critical: Not only are the morbidity and mortality disparities large (Lengerich et al. 2005; Rural Health Reform Policy Research Center 2014; Singh et al. 2012), but a substantial proportion of the US population still resides in rural areas. The Isolation scale will be a useful tool in the struggle to diminish such rural health disparities.

\section{References}

Alasia, A., Penney, C., Guimond, E., Bédard, F., and Bélanger, J. (2017). Measuring remoteness and accessibility: A set of indices for Canadian communities. Reports on Special Business Projects. MeasRemote2017.

American Lung Association (2012). Cutting tobacco's rural roots: Tobacco use in rural communities. http://www.lung.org/our-initiatives/research/lung-health-disparities/tobaccoin-rural-communities.html.

Bank, T. W. (2016). Measuring rural access: using new technologies. Number 107996. http://documents.worldbank.org/curated/en/367391472117815229/Measuringrural-access-using-new-technologies.

Burnham, K. and Anderson, D. (2002). Information and Likelihood Theory: A Basis for Model Selection and Inference, pages 49-97. Springer, New York, NY.

Byun, S., Meece, J. L., and Irvin, M. J. (2012). Ruralnonrural disparities in postsecondary educational attainment revisited. American educational research journal, 49(3).

Caldwell, J. T., Ford, C. L., Wallace, S. P., Wang, M. C., and Takahashi, L. M. (2016). Intersection of living in a rural versus urban area and race/ethnicity in explaining access to health care in the united states. American Journal of Public Health, 106(8):1463-1469. 
Centers for Disease Control and Prevention (2018). State Tobacco Activities Tracking and Evaluation (STATE) System. https://chronicdata.cdc.gov/health-area/tobacco-use.

Cromartie, J. B. and Bucholtz, S. (2008). Defining the "Rural" in Rural America. https://www.ers.usda.gov/amber-waves/2008/june/defining-the-rural-in-rural-america/.

DeVellis, R. F. (2011). Scale Development: Theory and Applications. SAGE Publications, Inc, 3 edition edition.

Doogan, N. J., Roberts, M. E., Wewers, M. E., Stanton, C. A., Keith, D. R., Gaalema, D. E., Kurti, A. N., Redner, R., Cepeda-Benito, A., Bunn, J. Y., and et al. (2017). A growing geographic disparity: Rural and urban cigarette smoking trends in the united states. Preventive Medicine, (104):79-85.

Ely, D. M., Driscoll, A. K., and Mathews, T. J. (2017). Infant mortality rates in rural and urban areas in the United States, 2014. Number NCHS data brief, no. 285. IMUS2017.

Esri (2017). A-16 Federally Derived Data: Open Data Catalog. http://openfedmaps.opendata.arcgis.com/.

Federal Communications Commission (2015). Residential Fixed Connections over 200 kbps per 1,000 Households by Census Tract. https://www.fcc.gov/reportsresearch/maps/residential-fixed-connections-over-200-kbps-december-2015/.

FedEx (2018). FedEx Service Guide.

Hart, L. G., Larson, E. H., and Lishner, D. M. (2005). Rural definitions for health policy and research. American Journal of Public Health, 95(7):1149-1155.

Hartley, D. (2004). Rural health disparities, population health, and rural culture. American Journal of Public Health, 94(10):1675-1678.

Humphreys, J. S., McGrail, M. R., Joyce, C. M., Scott, A., and Kalb, G. (2012). Who should receive recruitment and retention incentives? improved targeting of rural doctors using medical workforce data. Australian Journal of Rural Health, 20(1):310. Humphreys2012.

Humphreys, J. S. and Solarsh, G. (2008). Populations at Special Health Risk: Rural Populations, page 242253. Academic Press. SpclHealthRisk2008.

LaVeist, T. A., Gaskin, D., and Richard, P. (2011). Estimating the economic burden of racial health inequalities in the united states. International Journal of Health Services, 41(2):231238. LaVeist2011.

Lengerich, E. J., Tucker, T. C., Powell, R. K., Colsher, P., Lehman, E., Ward, A. J., Siedlecki, J. C., and Wyatt, S. W. (2005). Cancer incidence in kentucky, pennsylvania, and west virginia: Disparities in appalachia. The Journal of Rural Health, 21(1):39-47.

Li, Y., Long, H., and Liu, Y. (2015). Spatio-temporal pattern of chinas rural development: A rurality index perspective. Journal of Rural Studies, 38:1226. RuralChina2015.

Liese, A. D., Hibbert, J. D., Ma, X., Bell, B. A., and Battersby, S. E. (2014). Where are the food deserts? an evaluation of policy-relevant measures of community food access in south carolina. Journal of hunger \&ु environmental nutrition, 9(1):1632. Liese2014. 
McMillen, R., Breen, J., and Cosby, A. G. (2004). Rural-urban differences in the social climate surrounding environmental tobacco smoke: A report from the 2002 social climate survey of tobacco control. The Journal of Rural Health, 20(1):7-16.

Morrill, R., Cromartie, J., and Hart, G. (1999). Metropolitan, urban, and rural commuting areas: Toward a better depiction of the united states settlement system. Urban Geography, 20(8):727-748.

Multi-Resolution Land Characteristics Consortium (2014). National Land Cover Database2011. https://www.mrlc.gov/nlcd11_data.php.

National Academies of Sciences, Engineering, and Medicine (2016). Rationalizing Rural Area Classifications for the Economic Research Service: Workshop Summary. G. S. Wunderlich, Rapporteur, Comittee on National Statistics.

National Oceanic and Atmospheric Administration (2010). Global Radiance Calibrated Nighttime Lights. https://www.ngdc.noaa.gov/eog/dmsp/download_radcal.html.

Northridge, M. E., Vallone, D., Xiao, H., Green, M., Blackwood, J. W., Kemper, S. E., Duke, J., Watson, K. A., Burrus, B., and Treadwell, H. M. (2008). The importance of location for tobacco cessation: Rural-urban disparities in quit success in underserved west virginia counties. The Journal of Rural Health, 24(2):106-115.

OpenStreetMap contributors (2017). Planet dump retrieved from https://planet.osm.org. OSM.

Penchansky, R. and Thomas, J. W. (1981). The concept of access: Definition and relationship to consumer satisfaction. Medical Care, 19(2):127-140.

Roberts, M. E., Doogan, N. J., Kurti, A. N., Redner, R., Gaalema, D. E., Stanton, C. A., White, T. J., and Higgins, S. T. (2016). Rural tobacco use across the united states: How rural and urban areas differ, broken down by census regions and divisions. Health $\&$ Place, 39:153-159.

Rural Health Reform Policy Research Center (2014). The 2014 Update of the RuralUrban Chartbook. Retreived from https://ruralhealth.und.edu/projects/health-reformpolicy-research-center/pdf/2014-rural-urban-chartbook-update.pdf.

Scheil-Adlung, X. (2015). Global evidence on inequities in rural health protection: New data on rural deficits in health coverage for 174 countries. Number ESS Document No. 47. GlobalEvidenceRuralHealth.

Singh, G. K., Williams, S. D., Siahpush, M., Mulhollen, A., Singh, G. K., Williams, S. D., Siahpush, M., and Mulhollen, A. (2012). Socioeconomic, rural-urban, and racial inequalities in us cancer mortality: Part I-all cancers and lung cancer and part II-colorectal, prostate, breast, and cervical cancers. Journal of Cancer Epidemiology, Journal of Cancer Epidemiology, 2011, 2011:e107497.

S.J. Consulting Group, Inc. (2011). Measuring the benefits of rural postal service. Prepared for Postal Regulatory Commission (PRC). Retrieved from https://www.prc.gov/sites/default/files/archived/Rural_Service_Report.pdf. 
Sohi, I., Bell, B. A., Liu, J., Battersby, S. E., and Liese, A. D. (2014). Differences in food environment perceptions and spatial attributes of food shopping between residents of low and high food access areas. Journal of nutrition education and behavior, 46(4):241249. Sohi2014.

Substance Abuse and Mental Health Services Administration (2013). Results from the 2012 National Survey on Drug Use and Health: Summary of National Findings.

US Centers for Disease Control and Prevention (CDC) (2016). Linked Birth / Infant Death Records 2016, as compiled from data provided by the 57 vital statistics jurisdictions through the Vital Statistics Cooperative Program, on CDC WONDER Online Database. https://www.cdc.gov/nchs/pressroom/sosmap/infant_mortality_rates/infant_mortality.htm.

US Department of Agriculture Rural Development (2016a). Factsheet: Rural Business Development Grants. https://www.rd.usda.gov/files/fact-sheet/RD-FactSheet-RBS-RBDG.pdf.

US Department of Agriculture Rural Development (2016b). Factsheet: Rural Business Investment Program. https://www.rd.usda.gov/files/fact-sheet/RD-Factsheet-RBSRBusInvestmentProgram.pdf.

US Department of Agriculture (USDA) Economic Research Service (ERS) (2015a). Food Access Resource Atlas. https://www.ers.usda.gov/data-products/food-access-research-atlas/.

US Department of Agriculture (USDA) Economic Research Service (ERS) (2015b). Frontier and remote area codes. https://www.ers.usda.gov/data-products/frontier-and-remote-areacodes/frontier-and-remote-area-codes/\#2010 Frontier and Remote Area Codes Data Files.

US Department of Agriculture (USDA) Economic Research Service (ERS) (2017). Usda ers - geography of poverty. https://www.ers.usda.gov/topics/rural-economy-population/ruralpoverty-well-being/geography-of-poverty.aspx.

US Department of Health and Human Services (2016). Hrsa data warehouse - area health resource file 2015-2016 release. https://datawarehouse.hrsa.gov/topics/ahrf.aspx.

\footnotetext{
Affiliation:

Nathan J. Doogan

The Ohio State University

Columbus, Ohio

E-mail: doogan.1@osu.edu

URL: http://doogan.us/
}

\section{SocArXiv Website SocArXiv Preprints}

Social Science \& Medicine

doi.org/10.1016/j.socscimed.2018.09.005 https://socopen.org/

https://osf.io/preprints/socarxiv

Submitted: 2018.07.26

Accepted: 2018.09.06 